
JNM
J Neurogastroenterol Motil, Vol. 22 No. 1 January, 2016
pISSN: 2093-0879 elSSN: 2093-0887
http://dx.doi.org/10.5056/jnm 15130

\title{
Effects of Metoclopramide on Esophageal Motor Activity and Esophagogastric Junction Compliance in Healthy Volunteers
}

\author{
Hironobu Mikami, ${ }^{1 *}$ Norihisa Ishimura, ${ }^{1}$ Kousuke Fukazawa, ${ }^{2}$ Mayumi Okada, ${ }^{1}$ Daisuke Izumi, ${ }^{1}$ Shino Shimura, ${ }^{1}$ Eiko Okimoto, ${ }^{1}$ \\ Masahito Aimi, ${ }^{1}$ Shunji Ishihara, ${ }^{1}$ and Yoshikazu Kinoshita ${ }^{1}$ \\ ${ }^{1}$ Second Department of Internal Medicine, Shimane University Faculty of Medicine, Izumo, Japan; and ${ }^{2}$ Department of Gastroenterology and \\ Hepatology, Kasai City Hospital, Kasai, Japan
}

\section{Background/Aims}

Prokinetic drugs such as metoclopramide are frequently used as second-line therapy for patients with gastroesophageal reflux disease. However, their beneficial effects remain unclear. Esophageal motor activities and compliance of the esophagogastric junction (EGJ) are important for prevention of gastroesophageal reflux. Although metoclopramide has been reported to increase lower esophageal sphincter (LES) pressure, its effects on EGJ compliance have not been evaluated. In the present study, we investigated the effects of metoclopramide on esophageal motor activities and EGJ compliance.

\section{Methods}

Nine healthy male volunteers without abdominal symptoms were enrolled. Peristaltic esophageal contractions and LES pressure were examined using high-resolution esophageal manometry, while EGJ compliance was evaluated with an endoluminal functional lumenimaging probe. After obtaining baseline values for esophageal motor activities and EGJ compliance, metoclopramide (10 mg) was intravenously administered, then all measurements were repeated at 15 minutes after administration in each subject.

\section{Results}

Following administration of metoclopramide, mean resting LES pressure was significantly increased as compared with the baseline $(13.7 \pm 9.2$ vs $26.7 \pm 8.8 \mathrm{mmHg}, P<0.05)$. In addition, metoclopramide significantly augmented peristaltic contractions, especially in the distal esophageal segment $(P<0.05)$. On the other hand, distensibility index did not change after administration $(4.5 \pm 0.5$ vs $4.1 \pm 0.5 \mathrm{~mm}^{2} / \mathrm{mmHg}$ ), suggesting no significant effect of metoclopramide on EGJ compliance.

\section{Conclusions}

Metoclopramide augmented esophageal contractions without changing EGJ compliance in healthy adults.

(J Neurogastroenterol Motil 2016;22:112-117)

\section{Key Words}

Esophageal sphincter, lower; Esophagogastric junction; Manometry; Metoclopramide; Motor Activity

Received: August 13, 2015 Revised: October 8, 2015 Accepted: October 18, 2015

(a) This is an Open Access article distributed under the terms of the Creative Commons Attribution Non-Commercial License (http://creativecommons. org/licenses/by-nc/4.0) which permits unrestricted non-commercial use, distribution, and reproduction in any medium, provided the original work is properly cited.

${ }^{*}$ Correspondence: Hironobu Mikami, MD Second Department of Internal Medicine, Shimane University Faculty of Medicine 89-1, Enya-cho, Izumo, Shimane 693-8501, Japan

Tel: +81-853-20-2190, Fax: +81-853-20-2187, E-mail: d_h_m202@med.shimane-u.ac.jp 


\section{Introduction}

The patients with gastroesophageal reflux disease (GERD) suffer from heartburn and regurgitation, which are caused by reflux of stomach contents, leading to a significant impairment of quality of life. Proton pump inhibitor (PPI) administration is the current mainstay for first-line treatment of GERD because of the consistent inhibitory effect on acid secretion. However, use of a PPI alone is insufficient in approximately $30 \%$ of GERD patients. ${ }^{1}$ As secondline therapy, prokinetic drugs have been used in clinical settings, though their beneficial effects for GERD are not well elucidated. ${ }^{2,3}$

Metoclopramide, a prokinetic agent, has been widely used for treatment of gastrointestinal motility disorders. In addition to acting as a central dopamine $\mathrm{D}_{2}$ receptor antagonist, metoclopramide blocks the dopamine $\mathrm{D}_{2}$ receptor in peripheral nerve endings and promotes release of acetylcholine. As a result, metoclopramide augments gastrointestinal motility and enhances gastric emptying, and also increases lower esophageal sphincter (LES) tone. ${ }^{4-8}$

It has recently been reported that esophageal motor activities and compliance of the esophagogastric junction (EGJ) is important for prevention of gastroesophageal reflux, with the latter considered to cause greater volume reflux of gastric contents into the esophagus. $^{9-11}$ Fundoplication surgery for repairing the EGJ has been consistently shown to prevent pathological gastroesophageal reflux by restoration of normal EGJ compliance. .2,13 $^{13}$

An endo-luminal functional lumen imaging probe (EndoFLIP, Crospon Ltd, Galway, Ireland) has been developed as a novel device to determine cross-sectional area (CSA) and distensibility at the EGJ. ${ }^{9}$ We previously used this probe and high-resolution manometry (HRM) to show that high-dose mosapride (40 mg), a prokinetic agent that acts on the 5-hydroxytryptamine type 4-receptors, augments peristaltic contractions and mean resting LES pressure, which significantly reduced EGJ compliance, though the effects of mosapride on esophageal motor activity vary at lower doses. ${ }^{14,15}$

Although metoclopramide has been reported to increase LES pressure, its effects on EGJ compliance have not been evaluated. ${ }^{4-8}$ Therefore, the aim of the present study was to investigate the effects of metoclopramide on esophageal motor activities and EGJ compliance in healthy volunteers, for which we employed the EndoFLIP system and high-resolution 36-channel manometry.

\section{Materials and Methods}

\section{Subjects and Study Protocol}

Nine healthy male volunteers (mean age $25.4 \pm 3.1$ years) without chest or abdominal symptoms were enrolled in this study. None had a history of cardiac, respiratory, neurologic, or gastrointestinal disorders, or were taking any medications.

Peristaltic esophageal contractions and LES pressure were examined by high-resolution esophageal manometry (ManoScan360; Sierra Scientific Instruments, Los Angeles, CA, USA), while EGJ compliance was investigated using the EndoFLIP system. ${ }^{9}$ Prior to each measurement, an intravenous access was obtained. After baseline measurements of esophageal motor activities and EGJ compliance, $10 \mathrm{mg}$ of metoclopramide was administered as an intravenous single bolus. At 15 minutes after administration of metoclopramide, the measurements were repeated.

The study protocol was approved by the ethical committee of Shimane University Faculty of Medicine. Written informed consent was obtained from each subject before enrollment in the study, which was carried out in accordance with the Declaration of Helsinki. This study was registered with the University Hospital Medical Information Network clinical trials registry, number UMIN (000013521).

\section{Determination of Esophageal Motor Activity}

Esophageal motor activity was determined using highresolution 36-channel manometry, according to a previously reported method. ${ }^{14-16}$ Based on the manufacturer's instructions, the manometric catheter was inserted in a transnasal manner after local anesthesia (2\% lidocaine jelly) and measurements were performed in a supine position starting 5 minutes after insertion. Initially, we determined resting LES pressure for at least 5 minutes. Then, esophageal body peristaltic contractions were evaluated after a wet swallow of drinking water $(5 \mathrm{~mL})$. That was repeated at intervals of 2 minutes until 5 complete contraction records were obtained. Esophageal body peristaltic contractions were observed in 3 segments (proximal, middle, distal, segments 1, 2, 3, respectively), based on a previous report. ${ }^{14-16}$ LES pressure and peak peristaltic contraction pressure in the 3 segments of the esophageal body were analyzed using ManoView analysis software (Sierra Scientific Instruments). 


\section{Determination of Esophagogastric Junction Compliance}

EGJ compliance was determined by use of the EndoFLIP system. The probe was a $240-\mathrm{cm}$ long catheter with a $3-\mathrm{mm}$ outer diameter and equipped with a 14-cm long bag in the distal end, which is compliant to a maximal diameter of $25 \mathrm{~mm}$. Inside the bag, 16 paired impedance electrodes were placed at 4-mm intervals along with a pressure sensor. As the bag was filled with a specially formulated conductive solution, impedance across each segment was inversely proportional to the CSA of the bag at that locus. This allows the diameter and CSA of the bag to be measured, along with intra-bag pressure. Data from the 16 paired electrodes and pressure sensor were sampled by a recording unit, which displayed them in real time on a monitor. The catheter was calibrated based on the manufacturer's instructions, then inserted in a transnasal manner until the impedance electrodes straddled the EGJ at its mid-point. We ignored peristaltic waves, which were often visible. The bag was inflated to volumes of 20,30, and $40 \mathrm{~mL}$, with a 30 -second recording obtained at each distension volume. We evaluated minimum diameter (Dmin), narrowest CSA, and distensibility index (DI [mm/ $\mathrm{mmHg}]$ ) at each distension volume, as previously reported. EGJ distensibility index was defined as the narrowest CSA in relation to the corresponding intra-bag pressure and calculated using the following equation: narrowest CSA/intra-bag pressure + intragastric pressure offset.

\section{Statistical Methods}

Data are expressed as the mean $\pm \mathrm{SE}$. Statistical comparisons between metoclopramide administration and the baseline period were performed used a Wilcoxon signed rank test. Differences at $P<0.05$ were considered to be statistically significant. All analyses were performed using SPSS version 19.0 (IBM SPSS Japan Inc, Tokyo, Japan).

\section{Results}

All 9 subjects completed the study protocol without any adverse events. EndoFLIP data for 1 were not appropriately recorded due to technical problems, thus those obtained from 8 subjects were analyzed. In addition, data for esophageal motor activity obtained with HRM were successfully recorded in all cases.

Consistent with previous reports, mean resting LES pressure in a supine position was significantly elevated after intravenous ad-
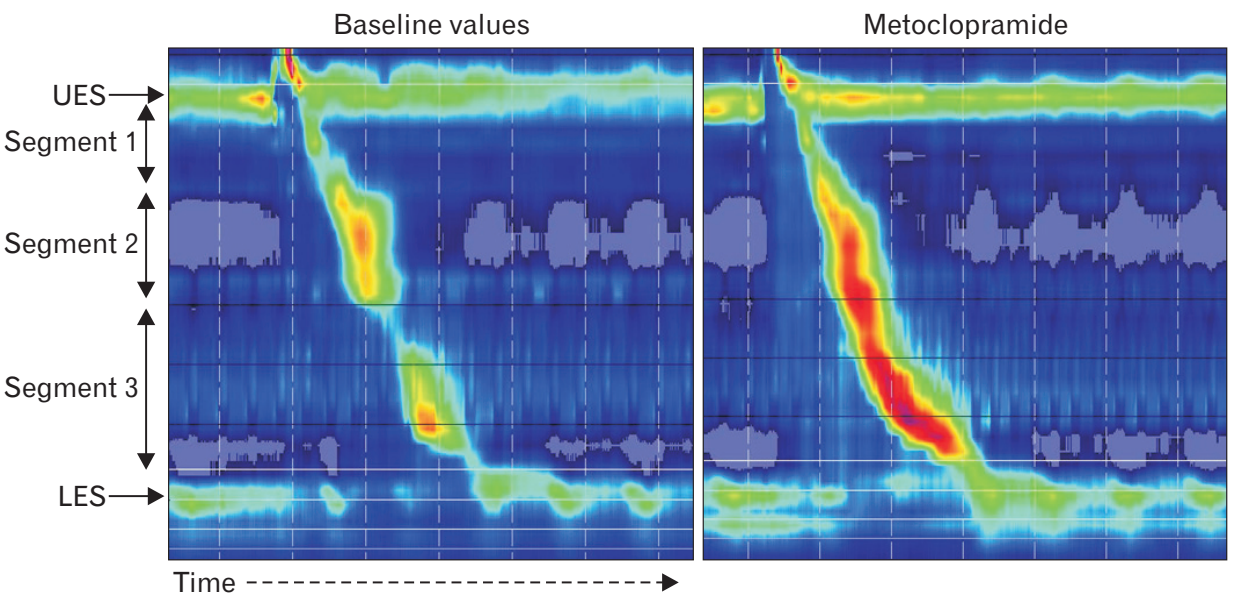

Figure 1. Esophageal motor activity with and without metoclopramide. Examination of a representative subject using high-resolution manometry. Esophageal peristaltic contractions (segments 1-3) and resting lower esophageal sphincter (LES) pressure were augmented after intravenous administration of metoclopramide. UES, upper esophageal sphincter.

Table 1. Esophageal Motor Activity

\begin{tabular}{lccc}
\hline & Baseline & Metoclopramide & $P$-value \\
Maximal peristaltic contraction $(\mathrm{mmHg})$ & & & \\
Segment 1 & $83.4 \pm 28.3$ & $86.9 \pm 25.6$ & $<.3 \pm 48.9$ \\
Segment 2 & $103.5 \pm 45.3$ & $114.3 \pm 0.5$ & $<0.05$ \\
Segment 3 & $109.8 \pm 45.4$ & $140.5 \pm 51.7$ & $<0.05$ \\
Resting LES pressure $(\mathrm{mmHg})$ & $13.7 \pm 9.2$ & $26.7 \pm 8.8$ & \\
\hline
\end{tabular}

LES, lower esophageal sphincter

Values are expressed as the mean $\pm \mathrm{SE}$. 
A

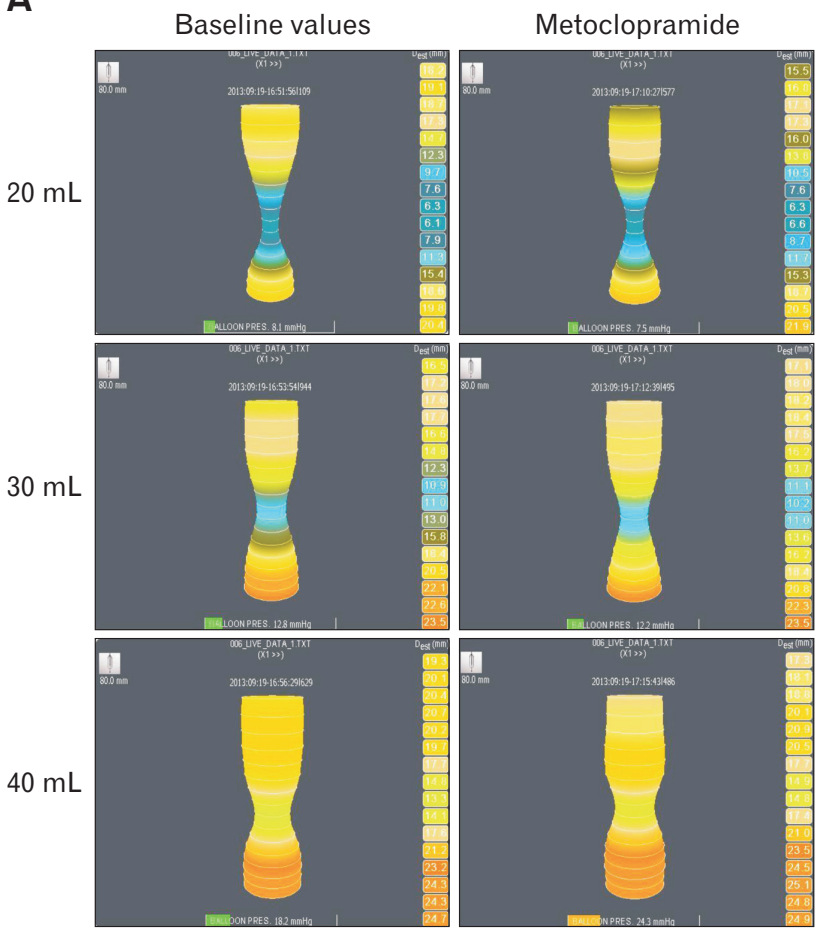

B

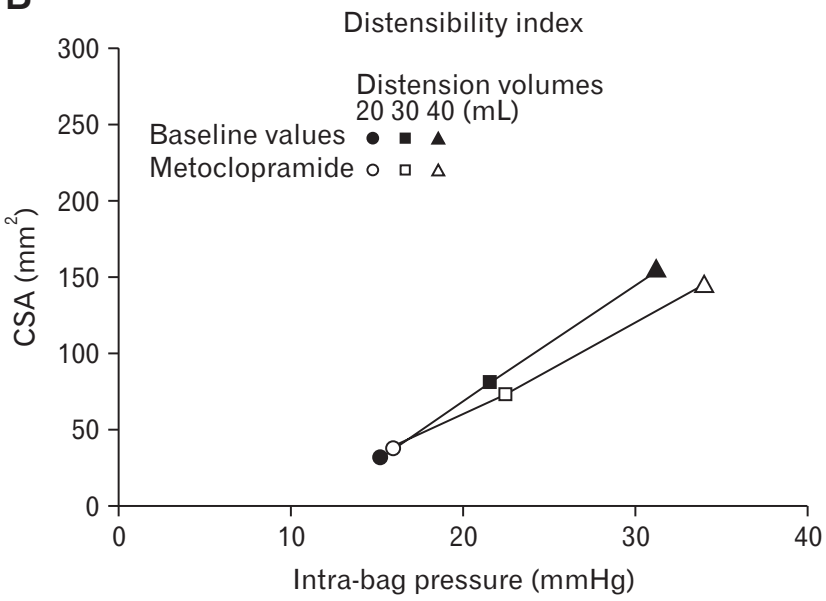

Figure 2. Esophagogastric junction (EGJ) compliance with and without metoclopramide. (A) EGJ compliance with and without metoclopramide administration. The EndoFILP bag was distended to 20,30 , and $40 \mathrm{~mL}$, which gave the bag an hourglass shape from the corresponding intra-bag pressure during both metoclopramide administration and the baseline period. There were no differences in bag shape following metoclopramide administration. (B) Distensibility index (DI) with and without metoclopramide. To determine the DI, intra-bag pressure (x-axis) and hiatal cross-sectional area (CSA; y-axis) were determined with the EndoFLIP bag distended to $20 \mathrm{~mL}$ (circles), $30 \mathrm{~mL}$ (squares), and $40 \mathrm{~mL}$ (triangles). There were no differences between the baseline period and the period following intravenous administration of metoclopramide. ministration of metoclopramide $(13.7 \pm 9.2$ vs $26.7 \pm 8.8 \mathrm{mmHg}$, $P<0.05)$. In addition, metoclopramide significantly augmented maximal peristaltic contractions, especially in segment 3 (distal; $109.8 \pm 45.4$ vs $140.5 \pm 51.7 \mathrm{mmHg}, P<0.05$ ) (Fig. 1 and Table 1). Contraction pressure in segments 1 and 2 (proximal and middle, respectively) also tended to be elevated after administration, though the differences did not reach statistical significance.

On the other hand, metoclopramide did not change EGJ compliance after intravenous administration. When the EndoFILP bag was distended to straddle the EGJ, the bag formed an hourglass shape by central contraction at the diaphragmatic hiatus during both metoclopramide administration and the baseline period (Fig. 2). There was no statistically significant differences in regard to hiatal minimum diameter $(13.8 \pm 1.5$ vs $13.6 \pm 0.7 \mathrm{~mm}$, distention volume $40 \mathrm{~mL}), \mathrm{CSA}\left(152.4 \pm 34.2\right.$ vs $146.1 \pm 15.3 \mathrm{~mm}^{2}$, distention volume $40 \mathrm{~mL}$ ), or distensibility index ( $4.5 \pm 0.5$ vs $4.1 \pm$ $0.5 \mathrm{~mm}^{2} / \mathrm{mmHg}$, distention volume $40 \mathrm{~mL}$ ) between the baseline period and following intravenous administration of metoclopramide (Table 2).

\section{Discussion}

GERD is defined as symptoms, such as heartburn and regurgitation, or esophageal mucosal injury produced by gastroesophageal reflux. A disorder of esophageal motor function is one of the important factors for occurrence of GERD. ${ }^{17}$ Notably, esophageal

Table 2. Esophagogastric Junction Compliance

\begin{tabular}{|c|c|c|}
\hline & Baseline & Metoclopramide \\
\hline \multicolumn{3}{|l|}{ Distention volume: $20 \mathrm{~mL}$} \\
\hline $\operatorname{Dmin}(\mathrm{mm})$ & $6.4 \pm 0.9$ & $6.9 \pm 1.6$ \\
\hline Hiatal CSA $\left(\mathrm{mm}^{2}\right)$ & $32.9 \pm 10.0$ & $39.4 \pm 18.5$ \\
\hline Intra-bag pressure (mmHg) & $15.5 \pm 5.1$ & $16.3 \pm 6.8$ \\
\hline $\mathrm{DI}\left(\mathrm{mm}^{2} / \mathrm{mmHg}\right)$ & $1.8 \pm 0.2$ & $1.9 \pm 0.2$ \\
\hline \multicolumn{3}{|l|}{ Distention volume: $30 \mathrm{~mL}$} \\
\hline $\operatorname{Dmin}(\mathrm{mm})$ & $10.2 \pm 1.9$ & $9.6 \pm 1.4$ \\
\hline Hiatal CSA $\left(\mathrm{mm}^{2}\right)$ & $84.5 \pm 33.2$ & $74.2 \pm 19.8$ \\
\hline Intra-bag pressure ( $\mathrm{mmHg}$ ) & $22.0 \pm 6.0$ & $22.4 \pm 7.1$ \\
\hline $\mathrm{DI}\left(\mathrm{mm}^{2} / \mathrm{mmHg}\right)$ & $3.5 \pm 0.6$ & $3.0 \pm 0.4$ \\
\hline \multicolumn{3}{|l|}{ Distention volume: $40 \mathrm{~mL}$} \\
\hline $\operatorname{Dmin}(\mathrm{mm})$ & $13.8 \pm 1.5$ & $13.6 \pm 0.7$ \\
\hline Hiatal CSA $\left(\mathrm{mm}^{2}\right)$ & $152.4 \pm 34.2$ & $146.1 \pm 15.3$ \\
\hline Intra-bag pressure (mmHg) & $31.2 \pm 6.8$ & $34.1 \pm 8.0$ \\
\hline $\mathrm{DI}\left(\mathrm{mm}^{2} / \mathrm{mmHg}\right)$ & $4.5 \pm 0.5$ & $4.1 \pm 0.5$ \\
\hline
\end{tabular}

Dmin, minimum diameter; CSA, cross-sectional area; DI, distensibility index. Values are expressed as the mean $\pm \mathrm{SE}$. 
body motor function and LES pressure are considered to be the most relevant factors for preventing gastroesophageal reflux. ${ }^{18,19}$ High LES pressure prevents reflux of gastric contents. In previous studies, low LES pressure and impaired esophageal peristalsis were observed in GERD patients, and free and stress-induced gastroesophageal refluxes are known to frequently occur in those cases. ${ }^{20-23}$ Furthermore, in order to clear gastric contents that have refluxed into the esophagus, efficient esophageal body peristaltic contractions are important. ${ }^{24-27}$

EGJ distensibility, an additional factor that prevents large volume gastroesophageal reflux, has recently drawn interest from researchers. Limited EGJ distensibility reduces large volume fluid reflux from the stomach and that in GERD patients has been reported to be augmented to a greater degree than that seen in healthy controls. ${ }^{9-11}$ Prokinetic agents are expected to improve those antireflux functions and considered to be effective for treatment of GERD. ${ }^{28}$ We previously reported that high-dose mosapride (40 $\mathrm{mg}$ ) not only augmented esophageal peristaltic contractions and mean resting LES pressure, but also reduced EGJ compliance, whereas a standard dosage did not consistently change esophageal motor activity. ${ }^{14,15}$

The present is the first study to show the effects of metoclopramide on esophageal motor activity and EGJ compliance in healthy adults using HRM and the EndoFLIP system. Metoclopramide is used as a prokinetic drug and has pharmacological actions similar to mosapride. Consistent with our previous study, metoclopramide was found to augment LES pressure to the same degree as mosapride. However, metoclopramide did not decrease EGJ distensibility, which was different from the effect of mosapride. Collectively, metoclopramide augmented esophageal motor activities, without affecting EGJ distensibility.

Considering our observations, it is important to note that the pharmacological characteristics of mosapride and metoclopramide are different, though both are used for gastrointestinal motility disorders as prokinetic agents. Mosapride mainly stimulates presynaptic $5-\mathrm{HT}_{4}$ receptors in the peripheral autonomic motor nervous system and augments acetylcholine release at the neuromuscular junction with augmented gastrointestinal motility. On the other hand, metoclopramide mainly inhibits presynaptic dopamine $\mathrm{D}_{2}$ receptors and increases acetylcholine release during neuromuscular reactions. These differences between the drugs may result in different effects on EGJ distensibility. In addition, mosapride has few effects on the central nervous system, as it has difficulty crossing the blood brain barrier. ${ }^{29}$ In contrast, metoclopramide can easily cross the blood brain barrier and blocks dopamine $\mathrm{D}_{2}$ receptors in the brain, including the brain stem. ${ }^{30,31}$ Therefore, the combined central and peripheral activities of metoclopramide may have different effects on EGJ distensibility as compared to mosapride.

The relationship between EGJ compliance and LES pressure remains controversial. Pandolfino et $\mathrm{al}^{32}$ reported a weak negative relationship and concluded that basal LES pressure likely plays only a small role in determining EGJ distensibility. The EGJ is a complex structure that exists along with the LES, crural diaphragm, and clasp and sling fibers at the angle of His. Although, individual influences of prokinetic agents on factors such as those investigated in the present study are difficult to evaluate by EndoFLIP measurements alone, metoclopramide, and mosapride may have different effects. ${ }^{33}$

There are some limitations to our study. First, we administrated metoclopramide to healthy young volunteers and not GERD patients with impaired esophageal motor functions. In addition, we have intravenously administrated metoclopramide in this study. For evaluating the therapeutic value of metoclopramide for the treatment of GERD, the effect of oral administration need to be studied. Furthermore, the number of subjects enrolled was small and an additional larger study that includes GERD patients may be necessary in the future.

In conclusion, intravenous administration of metoclopramide augmented esophageal body peristalsis and resting LES pressure, without changing EGJ compliance.

\section{Financial support: None.}

\section{Conflicts of interest: None.}

Author contributions: Yoshikazu Kinoshita: study concept and design; Masahito Aimi and Hironobu Mikami: acquisition, analysis, and interpretation of data; Hironobu Mikami: drafting of the manuscript; Norihisa Ishimura and Yoshikazu Kinoshita: critical revision of the manuscript for important intellectual content; Masahito Aimi and Hironobu Mikami: statistical analysis; and Yoshikazu Kinoshita: study supervision.

\section{References}

1. Adachi K, Furuta K, Miwa H, et al. A study on the efficacy of rebamipide for patients with proton pump inhibitor-refractory non-erosive reflux disease. Dig Dis Sci 2012;57:1609-1617.

2. Miwa $\mathrm{H}$, Inoue $\mathrm{K}$, Ashida $\mathrm{K}$, et al. Randomised clinical trial: efficacy of the addition of a prokinetic, mosapride citrate, to omeprazole in the treatment of patients with non-erosive reflux disease - a double-blind, 
placebo-controlled study. Aliment Pharmacol Ther 2011;33:323-332.

3. Hsu YC, Yang TH, Hsu WL, et al. Mosapride as an adjunct to lansoprazole for symptom relief of reflux oesophagitis. Br J Clin Pharmacol 2010;70:171-179.

4. Dilawari JB, Misiewicz JJ. Action of oral metoclopramide on the gastrooesophageal junction in man. Gut 1973;14:380-382.

5. Howard FA, Sharp DS. Effect of metoclopramide on gastric emptying during labour. Br Med J 1973;1:446-448.

6. Brock-Utne JG, Rubin J, Welman S, Dimopoulos GE, Moshal MG, Downing JW. The action of commonly used antiemetics on the lower esophageal sphincter. Br J Anaesth 1978;50:295-298.

7. Baumann HW, Sturdevant AL, McCallum RW. L-Dopa inhibits metoclopramide stimulation of the lower esophageal sphincter in man. Dig Dis Sci 1979;24:289-295.

8. Salem MR, Bruninga KW, Dodlapatii J, Joseph NJ. Metoclopramide does not attenuate cricoid pressure-induced relaxation of the lower esophageal sphincter in awake volunteers. Anesthesiology 2008;109:806-810.

9. Kwiatek MA, Pandolfino JE, Hirano I, Kahrilas PJ. Esophagogastric junction distensibility assessed with an endoscopic functional luminal imaging probe (EndoFLIP). Gastrointest Endosc 2010;72:272-278.

10. Ghosh SK, Kahrilas PJ, Brasseur JG. Liquid in the gastroesophageal segment promotes reflux, but compliance does not: a mathematical modeling study. Am J physiol Gastrointest Liver Physiol 2008;295:G920G933.

11. Kahrilas PJ, Shi G, Manka M, Joehl RJ. Increased frequency of transient lower esophageal sphincter relaxation induced by gastric distention in reflux patients with hiatal hernia. Gatroenterology 2000;118:688-695.

12. Pandolfino JE, Curry J, Shi G, joehl RJ, Brasseur JG, Kahrilas PJ. Restoration of normal distensive characteristics of the esophagogastric junction after fundoplication. Ann Surg 2005;242:43-48.

13. Hoppo T, McMahon BP, Witteman BP, et al. Functional lumen imaging probe to assess geometric changes in the esophagogastric junction following endolumenal fundoplication. J Gastrointest Surg 2011;15:11121120 .

14. Fukazawa K, Furuta K, Adachi K, et al. Effects of mosapride on esophageal motor activity and esophagogastric junction compliance in healthy volunteers. J Gastroenterol 2014;49:1307-1313.

15. Koshino K, Adachi K, Furuta K, et al. Effects of mosapride on esophageal functions and gastroesophageal reflux. J Gastroenterol Hepatol 2010;25:1066-1071.

16. Morita T, Furuta K, Adachi K, et al. Effects of rikkunshito (TJ-43) on esophageal motor function and gastroesophageal reflux. J Neurogastroenterol Motil 2012;18:181-186.

17. Dodds WJ, Hogan WJ, Helm JF, Dent J. Pathogenesis of reflux esophagitis. Gastroenterology 1981;81:376-394.

18. Castell DO, Murray JA, Tutuian R, Orlando RC, Arnold R. Review article: the pathophysiology of gastro-oesophageal reflux disease - oesophageal manifestations. Aliment Pharmacol Ther 2004;20(suppl 9):14-25.
19. Boeckxstaens GE. Review article: the pathophysiology of gastro-oesophageal reflux disease. Aliment Pharmacol Ther 2007;26:149-160.

20. Scheurer U, Halter F. Lower esophageal sphincter in reflux esophagitis. Scand J Gastroenterol 1976;11:629-634.

21. Kahrilas PJ, Dodds WJ, Hogan WJ, Kern M, Arndorfer RC, Reece A. Esophageal peristaltic dysfunction in peptic esophagitis. Gastroenterology 1986;91:897-904.

22. Dodds WJ, Dent J, Hogan WJ, et al. Mechanism of gastroesophageal reflux in patients with reflux esophagitis. N Engl J Med 1982;307:15471552.

23. Dodds WJ. The pathogenesis of gastroesophageal reflux disease. AJR Am J Roentgenol 1988;151:49-56.

24. Ho SC, Chang CS, Wu CY, Chen GH. Ineffective esophageal motility is a primary motility disorder in gastroesophageal reflux disease. Dig Dis Sci 2002;47:652-656.

25. Wong WM, Lai KC, Hui WM, et al. Pathophysiology of gastroesophageal reflux diseases in Chinese - role of transient lower esophageal sphincter relaxation and esophageal motor dysfunction. Am J Gastroenterol 2004;99:2088-2093.

26. Chitkara DK, Fortunato C, Nurko S. Esophageal motor activity in children with gastro-esophageal reflex disease and esophagitis. J Pediatr Gastroenterol Nutr 2005;40:70-75.

27. Iwakiri K, Kawami N, Sano H, et al. Mechanism of excessive esophageal acid exporsure in patients with reflux esophagitis. Dig Dis Sci 2009;54: 1686-1692.

28. Furukawa N, Iwakiri R, Koyama T, et al. Proportion of reflux esophagitis in 6010 Japanese adults: prospective evaluation by endoscopy. J Gastroenterol 1993;34:441-444.

29. Matsumoto S, Tagawa M, Amejima $\mathrm{H}$, et al. Absorption, distribution and excretion of [carbonyl- ${ }^{14} \mathrm{C}$ ] mosapride citrate after a single oral administration in rats, dogs and monkeys. Arzneimittelforschung 1993; 43:1084-1094.

30. Herberg LJ, Wishart TB. Selective permeation of the blood-brain barrier as a cause of the anomalous properties of 'atypical' neuroleptics. Pharmacol Biochem Behav 1980;12:871-873.

31. Brouwers JR, Assies J, Wiersinga WM, Huizing G, Tytgat GN. Plasma prolactin levels after acute and subchronic oral administration of domperidone and of metoclopramide: a cross-over study in healthy volunteers. Clin Endocrinol 1980;12:435-440.

32. Pandolfino JE, Shi G, Trueworthy B, Kahrilas PJ. Esophagogastric junction opening during relaxation distinguishes nonhernia reflux patients, hernia patients, and normal subjects. Gastroenterology 2003;125:10181024 .

33. Tucker E, Sweis R, Anggiansah A, et al. Measurement of esophagogastric junction cross-sectional area and distensibility by an endoluminal functional lumen imaging probe for the diagnosis of gastroesophageal reflux disease. Neurogastroenterol Motil 2013;25:904-910. 